\title{
A Novel Measurement Using Digital Radiography to Minimize Fluoroscopy in Total Hip Arthroplasty
}

\author{
Andrew G. Yun, MD, MBA ${ }^{1}$ Marilena Qutami, PA-C ${ }^{1} \quad$ Kory B. Dylan Pasko, BS ${ }^{1}$ \\ ${ }^{1}$ Department of Orthopedic Surgery, Center for Hip and Knee \\ Replacement, Providence St. John's Health Center, Santa Monica, \\ California \\ Address for correspondence Kory B. Dylan Pasko, BS, Department \\ of Orthopedic Surgery, Center for Hip and Knee Replacement, \\ Providence St. John's Health Center, 2001 Santa Monica Blvd \#760, \\ Santa Monica, CA 90404 (e-mail: korypasko@g.ucla.edu). \\ J Hip Surg 2021;5:96-100.
}

\begin{abstract}
Preoperative templating for total hip arthroplasty (THA) is fraught with uncertainty. Specifically, the conventional measurement of the lesser trochanter to the center (LTC) of the femoral head used in preoperative planning is easily measured on a template but not measurable intraoperatively. The purpose of this study was to examine the utility of a novel measurement that is reproducible both on templating and in surgery as a more accurate and practical guide. We retrospectively reviewed 201 patients with a history of osteoarthritis who underwent primary THA. For preoperative templating, the distance from the top of the lesser trochanter to the equator (LeTE) of the femoral head was measured on a calibrated digital radiograph with a neutral pelvis. This measurement was used intraoperatively to guide the choice of the trial neck and head. As with any templating technique, the goal was to construct a stable, impingementfree THA with equivalent leg lengths and hip offset. In evaluating this novel templating technique, the primary outcomes measured were the number of trial reductions and the amount of fluoroscopic time, exposures, and radiation required to obtain a balanced THA reconstruction. Using the LeTE measurement, the mean number of trial reductions was 1.21 , the mean number of intraoperative fluoroscopy images taken was 2.63 , the mean dose of radiation exposure from fluoroscopy was $0.02 \mathrm{mGy}$, and the mean fluoroscopy time per procedure was 0.6 seconds. In hips templated with the conventional LTC prior to the LeTE, the mean fluoroscopy time was 0.9 seconds. There

\section{Keywords}

- total hip arthroplasty

- templating

- digital radiography

- fluoroscopy was a statistically significant difference in fluoroscopy time $(p<0.001)$. The LeTE is a reproducible measurement that transfers reliably from digital templating to surgery. This novel preoperative templating metric reduces the fluoroscopy time and consequent radiation exposure to the surgical team and may minimize the number of trial reductions.
\end{abstract}

Preoperative templating in total hip arthroplasty (THA) is a crucial step for restoring proper balance and kinematics. ${ }^{1-4}$ Accurate templating, however, is limited by multiple uncertainties including leg position, pelvic rotation, and magnification. ${ }^{4,5}$ Consequently, surgeons struggle intraoperatively with key decisions based on ambiguous planning information. Other than gross clinical markers of impingement or

received

August 18, 2020

accepted after revision

January 26, 2021

published online

June 15, 2021

stability testing through a range of motion, more precise measurements of leg lengths, offset, and implant position are difficult to gauge with precision during surgery.

Improvements in technology to reduce radiation exposure are leading the shift from plain films to digital radiography (DR). The historic methods of THA templating with standard 18 to $20 \%$ magnification acetate sheets, however, remain

(c) 2021. Thieme. All rights reserved. Thieme Medical Publishers, Inc., 333 Seventh Avenue, 18th Floor, New York, NY 10001, USA
DOI https://doi.org/ 10.1055/s-0041-1728760. ISSN 2472-8446. 
unchanged. By convention, the acetabular position is estimated, and then the distance from the top of the lesser trochanter to the center (LTC) of rotation is measured to guide intraoperative choices for head and neck length. ${ }^{1}$

Although the LTC is straightforward to measure on templates, it is not actually measurable intraoperatively with the trial femoral head present. The center of the head as seen on the acetate cannot be identified on the real trial femoral head. Instead, the actual intraoperative distance measured is the length from the top of the lesser trochanter to the equator (LeTE) of the femoral head trial. These two measurements are not the same, and this difference has clinical implications.

We propose a novel, alternative measurement for THA to guide preoperative and intraoperative templating based on the LeTE. Using advances in DR and programming, current software is able to calculate the "equator" of a specified head diameter and length. The computed distance from this clear landmark to a point on the lesser trochanter can be reproducibly measured intraoperatively. It is hypothesized, therefore, that having more accurate information will improve the accuracy of intraoperative decisions and, thereby, decrease the radiographic exposures used in THA.

\section{Materials and Methods}

We retrospectively reviewed a total of 201 consecutive patients who underwent primary THA with DR. An image intensifier was connected into the DR system to measure intraoperative images. Surgery was performed by a single surgeon at a single institution with a minimum of 3 months follow-up. The goal of surgery was to construct a stable, impingement-free THA with equal hip length and symmetric offset compared with the nonoperative side, as previously reported by Matta et al and others. ${ }^{6-8}$ As a proxy for accuracy in templating, the primary outcome measures were the number of trial reductions needed, the number of fluoroscopic images taken during surgery, the fluoroscopic exposure time (seconds), and the radiation dose (mGy) needed intraoperatively to achieve a symmetric reconstruction. For comparison, the fluoroscopy time per procedure was contrasted to a control group of 83 THAs templated with the LTC. In the control group, the number of trial reductions, the number of fluoroscopic images, and the radiation dose were not available.

\section{Digital Radiography Templating}

Templating was performed in the operating room prior to incision to maximize accuracy. A 25-mm calibrated marker was placed between the legs at the estimated level of the femur. Sequential digital radiographs were then taken of the low anteroposterior (AP) pelvis with incremental changes in positioning until symmetry was confirmed. For pelvic symmetry, the sacrum was aligned with the pubic symphysis, the obturator foramen were of equal size, and the radiographic teardrops were symmetric. For the bilateral femurs, the hips were symmetrically rotated to a neutral position based on the size and appearance of the greater and lesser trochanters. The legs were abducted to the same angle (-Fig. $\mathbf{1}$ ).

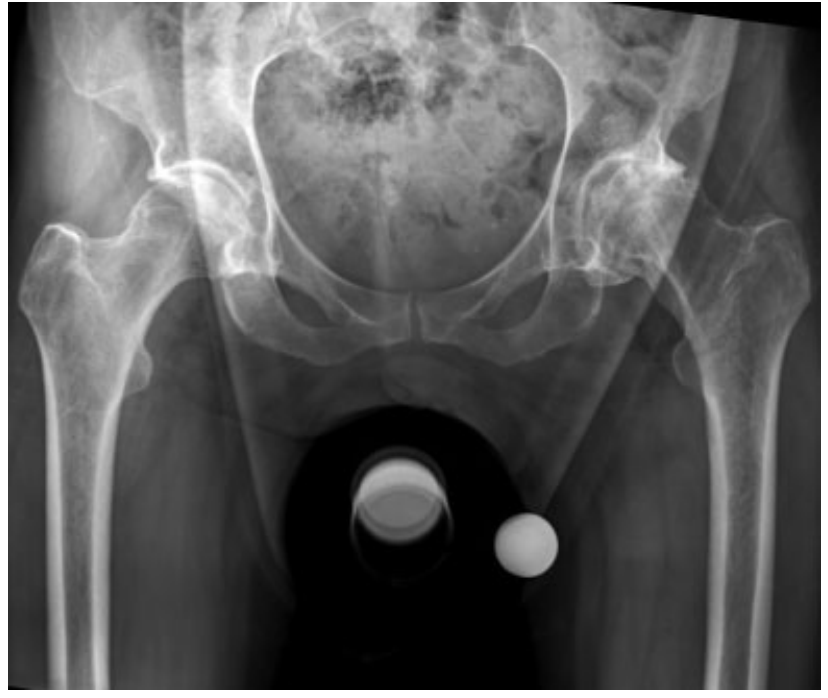

Fig. 1 Anteroposterior pelvic radiograph taken under anesthesia prior to surgery. A metal ball at the level of the hip is used for calibration. Pelvic symmetry is evaluated by the symmetries of the obturator foramen, the radiographic teardrops, and the alignment of the pubic symphysis with the spine. Femoral symmetry is confirmed by symmetric abduction, rotation, and position of the lesser trochanters.

Once a radiograph with ideal symmetry was obtained, the image was transferred to the Radlink software program for templating (El Segundo, CA). The radiograph was calibrated exactly to the 25-mm marker. Leg length discrepancy was determined by comparing the vertical distance from a similar point on each of the lesser trochanters to a horizontal line drawn across the bottom of each radiographic teardrop. Offset was calculated as the horizontal distance from the same point on the lesser trochanters to the most lateral edge of the ischium. The cup size, position, and center of rotation were templated in the standard fashion. The femur stem size and height were based on the best fit and fill of the template in relation to the femoral canal in the standard fashion.

The Radlink software was then used to create a virtual circumference around the templated femoral head based on the anticipated diameter of the real head, typically 32 or $36 \mathrm{~mm}$. A digital diameter was applied across the templated head at a perpendicular angle to the femoral neck. The point where the diameter met the circumference was established as the "equator." The distance from the top of the lesser trochanter to the equator was then defined as the LeTE. Importantly, the position of this point will vary with changes in head diameter, head length, stem neck length, stem neck offset, and stem neck angle. For example, a 135-degree neck shaft angle will have a different LeTE than a 125-degree neck angle with the same head. Similarly, a neutral femoral head trial will have a different LeTE than a minus femoral head trial (-Fig. 2). Furthermore, the length of the LeTE measurement calculated two-dimensionally on the DR will vary based on the rotation of the femur. Hence, a preoperative radiograph with a neutral pelvis and symmetrically positioned hips is a necessary condition for accuracy in planning. 


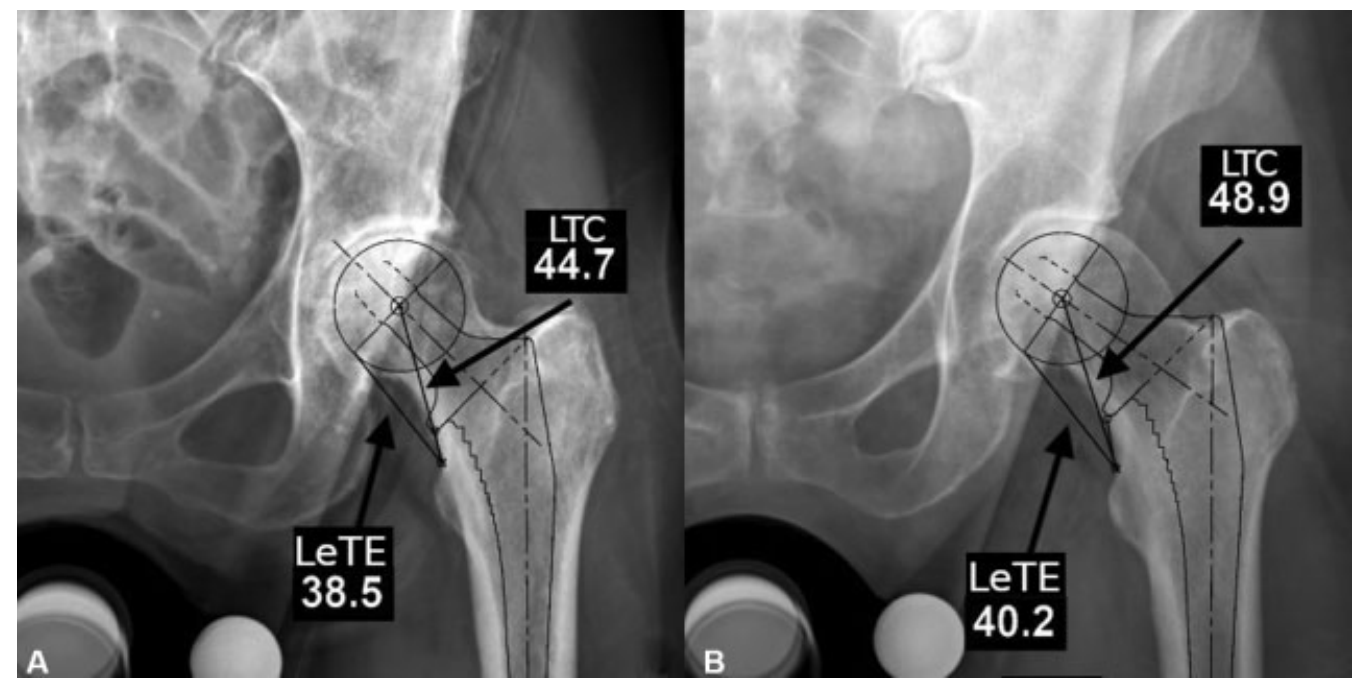

Fig. 2 (A, B) Preoperative templating for a 135- and 125-degree neck angle. (A) The LeTE is $38.5 \mathrm{~mm}$ and the LTC is $44.7 \mathrm{~mm}$ with a 135 -degree neck angle. (B) The LeTE is $40.2 \mathrm{~mm}$ and the LTC is $48.9 \mathrm{~mm}$ with a 125-degree neck angle. LeTE, lesser trochanter to the equator; LTC, lesser trochanter to the center.

\section{Surgical Technique}

The direct anterior approach (DAA) for THA was similar to that previously described by Matta et al. ${ }^{6}$ One fluoroscopic image was taken for positioning of the acetabular implant. Once the trial neck and head were placed, a ruler was used to measure the distance from the lesser trochanter to the equatorial marking of the head trial. Adjustments to the trial components were then made so that the intraoperative ruler measurement matched the LeTE calculated on the digital software (-Fig. 3). With the trials reduced, a second fluoroscopic image was then taken and transferred to the Radlink system for measurement of position, hip length, and offset. Intraoperative adjustments to stem sizing, stem height, stem angle, neck shaft angle, and head length were then made to correct any deviation from stability or symmetry. The total number of images, the number of trial reductions, and the

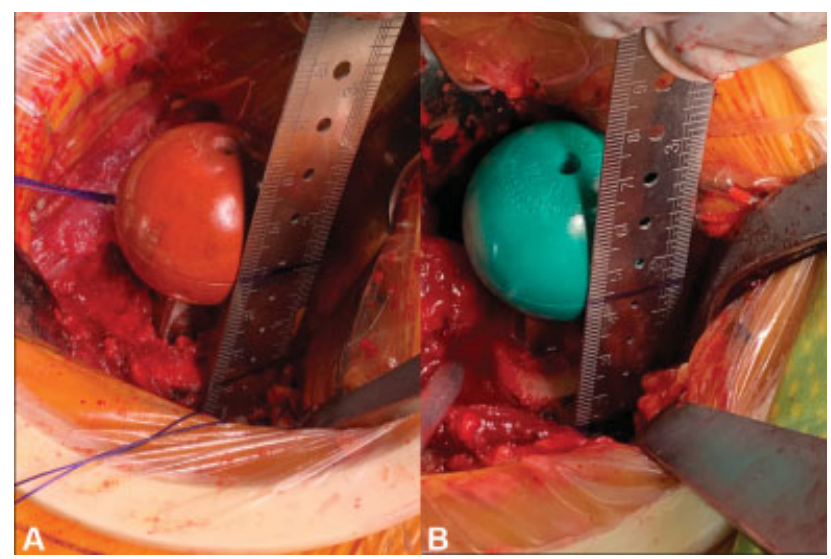

Fig. 3 (A, B) Intraoperative clinical measurements from the LeTE of the trial head. (A) The distance from the LeTE of the $-2 \mathrm{~mm}$ femoral head matches the preoperative distance from templating. The templated neck cut also matches. (B) Similarly, the LeTE is clinically reproduced with a neutral head in a different patient. LeTE, lesser trochanter to the equator. amount of radiation in mGy were then recorded in the index group. After the final fluoroscopic confirmation and stability check, the real implants were placed.

\section{Statistical Analysis}

The Mann-Whitney's $U$ test was chosen to determine statistical significance of the fluoroscopy time per procedure using the LeTE versus conventional LTC. The nature of the hypothesis testing was two-tailed. Statistical significance was determined at a $p$-value of less than 0.05 . Data were analyzed using IBM SSPS statistics software, version 25.0.0.

\section{Results}

Of the 284 DAA THAs performed, 201 patients underwent primary THA using the LeTE and 83 patients underwent primary THA using conventional measurement of the LTC. Using the LeTE, the mean number of intraoperative fluoroscopy images taken was 2.63 (range, 2-6). As a matter of protocol, one image is taken initially for acetabular cup positioning, and subsequent images are taken with the femoral trials in place until a balanced reconstruction is obtained. The mean fluoroscopy time prior to the LeTE was 0.9 seconds (standard deviation $[\mathrm{SD}] \pm 0.0065$ ) (range, 0.6-1.8), and the mean fluoroscopy time using the LeTE was 0.6 seconds ( $\mathrm{SD} \pm 0.0007$ ) (range, 0.6-1.2). There was a statistically significant difference in fluoroscopy time, $p<0.001$. Using the LeTE, the mean dose of radiation exposure per procedure from fluoroscopy was $0.02 \mathrm{mGy}$ (range, $0.01-0.21$ ) and the mean number of trial reductions was 1.21 (range, 1-3). Of the 201 cases performed using the LeTE, there were 161 cases with 1 trial reduction (80\%), 38 cases with 2 trial reductions (19\%), and 2 cases with 3 trial reductions (1\%). For the cohort of 83 THAs prior to implementation of the LeTE, the number of trial reductions and dose of radiation exposure were not available. 


\section{Discussion}

For several reasons, prior to the advent of computerized digital templating, accurate templating was hindered and measurements such as the LeTE were difficult to obtain. The hip radiographs obtained in the office were far more helpful for diagnosis that for surgical planning. Historical methods of THA templating on film radiographs were limited to landmarks that were reproducible. Consequently, the convention of using the radiograph to find the distance from the lesser trochanter to the center of the head, known as the LTC, became the best approximation. ${ }^{1}$

However, while a helpful estimate, the LTC does not correspond to what is actually measured in surgery. It is important not to confuse the intraoperative measurement between the lesser trochanter and trial head equator with the radiographic measurement of LTC. They are, in fact, two different measurements, and following the LTC measurement will result in a longer construct than intended.

Previously, the magnification of a radiographic film could vary from 105 to $120 \%{ }^{4,5}$ The acetate templates used to measure these radiographs were only available at a specific magnification of $117 \%$. The hope was that film and template magnification were sufficiently similar to create intraoperative accuracy. With the advent of DR and improved software, however, greater precision and accuracy are now possible. The sizing of bone and templates can now be calibrated to the size of a known marker. ${ }^{4}$

Another source of templating error arises from measurements taken on an AP pelvic radiograph without accounting for pelvic or femoral rotation. Often, the external rotation contractures of the arthritic hip create the appearance of a relative valgus femur with reduction of offset. Instant digital images now available with DR and small positioning adjustments of the pelvis and hips made preoperatively with the patient on the operating table optimize the radiograph used for surgical planning. ${ }^{4}$

Finally, prior to DR templating, the acetate templates could only identify the center of a given head length, and not the point at the circumference of a specific head diameter nor its corresponding change in position based on length. Currently, a virtual circumference of any diameter $(28,32,36$, or $40 \mathrm{~mm})$ can be drawn around a specified center of neck or head length, and a point on the circumference perpendicular to the neck shaft angle can be identified. The distance from this point to the lesser trochanter is easily measured both on DR and in surgery. Similar to the power of a spreadsheet that allows one to run multiple iteration of data scenarios effortlessly, the instantaneous ability to modify head size, head length, and neck shaft angle on a digital template creates far more opportunities to compare preoperative plans than a wax marker and acetate sheet.

Using this measurement has helped reduce the number of trial reductions and the corresponding number of intraoperative images needed to create the appropriate construct. Prior to the LeTE, it was common to require multiple reductions and images until the intraoperative images demonstrated equivalent leg length and offset. Currently, it is possible to achieve the desired reconstructive target with one trial reduction $80 \%$ of the time. Additionally, fewer reductions also reduce the amount of fluoroscopic radiation needed during surgery. As an issue of radiation stewardship, we report a mean fluoroscopy time of 0.6 seconds, compared with other studies that report a mean fluoroscopy time of 9.6 to 49.8 seconds per procedure as seen in - Table 1. ${ }^{9-14}$ While reducing X-ray exposure times in this series is not wholly related to the use of the LeTE measurement, it is an important aspect of the overall process of incrementally minimizing radiation exposure to the surgical team.

There are several limitations to this study. One major limitation is that it is a single surgeon's small retrospective

Table 1 Comparison with previous studies on direct anterior approach total hip arthroplasty fluoroscopy time and radiation exposure per procedure

\begin{tabular}{|c|c|c|c|c|}
\hline Study & Mean fluoroscopy time (s) & Mean radiation exposure (mGy) & Radiation exposure recorded by & $N$ \\
\hline Curtin et al $^{9}$ & 23.74 & 2.97 & Fluoroscopy unit & 157 \\
\hline \multicolumn{5}{|l|}{ Pomeroy et $\mathrm{al}^{10}$} \\
\hline Surgeon 1 & 18.51 & 2.396 & Helmet mounted & 30 \\
\hline Surgeon 2 & 15.63 & 2.139 & Dosimeter & 30 \\
\hline Surgeon 3 & 11.06 & 1.462 & & 30 \\
\hline Jinnai et $\mathrm{a}^{11}$ & 49.8 & & & 313 \\
\hline McArthur et al ${ }^{12}$ & 35.4 & & & 51 \\
\hline \multicolumn{5}{|l|}{ McNabb et al ${ }^{13}$} \\
\hline Surgeon 1 & 9.6 & 1.24 & Fluoroscopy unit & 18 \\
\hline Surgeon 2 & 21.8 & 2.96 & & 12 \\
\hline Surgeon 3 & 11.06 & 1.48 & & 15 \\
\hline Weeks et al ${ }^{14}$ & 28.65 & & & 50 \\
\hline Yun et al & 0.6 & 0.02 & Fluoroscopy unit & 201 \\
\hline
\end{tabular}


series. With a single surgeon there is an intrinsic risk of bias, and so we have tried to rely on objective points of reference such as fluoroscopy time, radiation dose, and number of trial reductions. Additionally, while we have found the LeTE to be helpful, we can only demonstrate indirectly that it was more reliable than the LTC using the relative decrease in intraoperative fluoroscopy time. It is at best difficult to calibrate the level of effort required to achieve accuracy during surgery using the proxy of these quantitative measures. Another major limitation is that in our control group, which consisted of 83 cases performed prior to the implementation of the LeTE, we did not have the foresight to record measurements of radiation exposures or the number of trial reductions. Technologically, it is also possible that traditional acetate templates modified to show the equator of the femoral head trial could be a reliable substitute for the software program embedded in the DR platform. Future comparative studies with technology more advanced than fluoroscopy, such as computer navigation or robotic-assisted THA, may yield improved results and potentially remove the need for intraoperative imaging altogether.

\section{Conclusion}

Ongoing advances in radiographic software and technology warrant advances in templating and measurement as well. Conventional estimates such as the LTC may be superseded by information previously unavailable prior to the advent of enhanced software with DR. Preoperative measurements that directly correspond to the intraoperative measurement are likely to be far more helpful during surgery. Until there is a wider adoption of this measurement, further investigation using the LeTE is warranted.

\section{Funding}

This research did not receive any specific grant from funding agencies in the public, commercial, or not-forprofit sectors.

\section{Conflict of Interest \\ None declared.}

\section{References}

1 Della Valle AG, Padgett DE, Salvati EA. Preoperative planning for primary total hip arthroplasty. J Am Acad Orthop Surg 2005;13 (07):455-462

2 Shaarani SR, McHugh G, Collins DA. Accuracy of digital preoperative templating in 100 consecutive uncemented total hip arthroplasties: a single surgeon series. J Arthroplasty 2013;28(02): 331-337

3 Eggli S, Pisan M, Müller ME. The value of preoperative planning for total hip arthroplasty. J Bone Joint Surg Br 1998;80(03):382-390

4 Wang Q Xiao J, Zhu L, et al. Acetate templating on calibrated standing digital radiograph improves accuracy of preoperative templating for total hip arthroplasty. Orthop Traumatol Surg Res 2017;103(03):341-347

5 Gorski JM, Schwartz L. A device to measure X-ray magnification in preoperative planning for cementless arthroplasty. Clin Orthop Relat Res 1986;(202):302-306

6 Matta JM, Shahrdar C, Ferguson T. Single-incision anterior approach for total hip arthroplasty on an orthopaedic table. Clin Orthop Relat Res 2005;441:115-124

7 Yun A, Qutami M, Pasko KBD. Salvage of failed femoral neck fracture fixation with conversion total hip arthroplasty using the direct anterior approach. Hip Pelvis 2020;32(04):199-206

8 Yun A, Qutami M, Pasko KBD. Conversion total hip arthroplasty with a direct anterior approach for failed treatment of acetabular fractures. J Hip Surg 2020;4:129-133

9 Curtin BM, Armstrong LC, Bucker BT, Odum SM, Jiranek WA. Patient radiation exposure during fluoro-assisted direct anterior approach total hip arthroplasty. J Arthroplasty 2016;31(06): 1218-1221

10 Pomeroy CL, Mason JB, Fehring TK, Masonis JL, Curtin BM. Radiation exposure during fluoro-assisted direct anterior total hip arthroplasty. J Arthroplasty 2016;31(08):1742-1745

11 Jinnai Y, Baba T, Zhuang X, et al. Does a fluoro-assisted direct anterior approach for total hip arthroplasty pose an excessive risk of radiation exposure to the surgeon? SICOT J 2020;6:6

12 McArthur BA, Schueler BA, Howe BM, Trousdale RT, Taunton MJ. Radiation exposure during fluoroscopic guided direct anterior approach for total hip arthroplasty. J Arthroplasty 2015;30(09): 1565-1568

13 McNabb DC, Jennings JM, Levy DL, Miner TM, Yang CC, Kim RH. Direct anterior hip replacement does not pose undue radiation exposure risk to the patient or surgeon. J Bone Joint Surg Am 2017;99(23):2020-2025

14 Weeks CA, Preston S, Howard JL, Vasarhelyi EM, Matz J, Lanting BA. Preoperative pelvic tilt analysis reduces fluoroscopy time in direct anterior total hip arthroplasty. Orthopedics 2016;39(05): e962-e966 